\title{
A Novel Approach to Generalized Intuitionistic Fuzzy Sets Based on Interpolative Boolean Algebra
}

\author{
Pavle Milošević *(D), Bratislav Petrović $(\mathbb{D})$ and Ivana Dragović \\ Faculty of Organizational Sciences, University of Belgrade, Jove Ilića 154, 11000 Belgrade, Serbia; \\ bratislav.petrovic@fon.bg.ac.rs (B.P.); ivana.dragovic@fon.bg.ac.rs (I.D.) \\ * Correspondence: pavle.milosevic@fon.bg.ac.rs; Tel.: +381-11-3950-852
}

Citation: Milošević, P.; Petrović, B.; Dragović, I. A Novel Approach to Generalized Intuitionistic Fuzzy Sets Based on Interpolative Boolean Algebra. Mathematics 2021, 9, 2115. https://doi.org/10.3390/math9172115

Academic Editor: Basil Papadopoulos

Received: 9 June 2021

Accepted: 28 July 2021

Published: 1 September 2021

Publisher's Note: MDPI stays neutral with regard to jurisdictional claims in published maps and institutional affiliations.

Copyright: () 2021 by the authors. Licensee MDPI, Basel, Switzerland. This article is an open access article distributed under the terms and conditions of the Creative Commons Attribution (CC BY) license (https:// creativecommons.org/licenses/by/ $4.0 /)$.

\begin{abstract}
One of the main issues in IFS theory are generalizations of intuitionistic fuzzy set (IFS) definition as well as IFS operations. In this paper, we present the LBIFS-IBA approach by applying operations based on interpolative Boolean algebra (IBA) on generalized IFS. Namely, LBIFS are defined as a special case of Liu's generalized IFS with the maximal interpretational surface. By extending the interpretational surface, the descriptive power of the approach is enhanced, and therefore the problematic situations when $\mu_{A}+v_{A}>1$ can be modeled. In addition, IBA-based algebra secures Boolean properties of the proposed approach. Considerable attention is given to comprehension of uncertainty within LBIFS-IBA, i.e., we propose a novel manner of uncertainty interpretation by treating values from $[-1,1]$ interval. In order to prove its importance, we compare LBIFS-IBA with several well-known IFS generalizations, showing that only our approach offers meaningful uncertainty interpretation is all selected cases. Additionally, we illustrate the practical benefits of LBIFS-IBA by applying it to an example of modeling Japanese candlesticks for price charting and paying special attention to uncertainty interpretation.
\end{abstract}

Keywords: LBIFS-IBA approach; generalization of intuitionistic fuzzy sets; IFS-interpretational triangle; interpolative Boolean algebra; uncertainty interpretation; Japanese candlestick modeling

\section{Introduction}

Intuitionistic fuzzy set (IFS) theory is a valuable tool for information presentation and manipulation [1,2]. IFSs are considered to be a generalization of traditional fuzzy sets since they include both a degree of membership and a degree of non-membership in data modeling. Furthermore, IFS may be seen as a powerful tool for expressing and handling non-determinacy (uncertainty). Classical operations and relations over IFSs extend the operations and relations over fuzzy sets [3]. Furthermore, there is a significant number of papers that deal with additional operations and algebras for IFS (e.g., [4-7]). Since IFSs are conceptualized as fuzzification of estimations in Brouwer's sense, Boolean-like algebras over IFS have not been the subject of extensive research. However, some papers deal with this idea [6,8]. On the other hand, the discussion and analysis of Boolean properties within conventional fuzzy logic is a well-studied topic, e.g., see [9-12].

The idea of challenging IFS definition and its restrictions formed one of the most inspirational areas of research within IFS theory. The main practical justification for proposing various types of generalized intuitionistic fuzzy sets (GIFS) is to avoid the need to alter the experts' estimations in real-world situations [2]. Subsequently, GIFS should maintain clearcut meaning and interpretation when solving practical problems. There are several main directions for generalizing the IFS definition. The first one refers to power and root-type GIFS [13-15]. Some authors try to extend the interpretational surface of GIFS by introducing a logical relationship between membership and non-membership degrees [16] or adding a constant to the upper boundary of the restriction [17]. The third direction of IFS generalizations considers uncertainty as an independent variable and 
assesses it separately from membership and non-membership (see $[18,19])$. Some of these approaches have proven to be valuable from both theoretical and practical points of view, e.g., [20-23]. However, it should be pointed out that the numerous propositions of GIFS with same/similar notion have generated terminological confusion [24]. Furthermore, there is an ongoing brisk debate on which approach should be considered as more general and if some of the approaches may be found as superficial [25-29].

Although most IFS generalizations are intuitive and mathematically correct, the question of applicability and interpretability of the proposed approaches arises. Most of the papers on this topic exclusively provide a mathematical apparatus for generalization without any explanation of why generalization is needed. Furthermore, most of the papers neither provide necessary interpretation nor the application guidelines.

This paper continues the work begun in $[7,30]$, where we studied the possibility of introducing operations inspired by interpolative Boolean algebra [31] (IBA) in IFS theory. Herein, we aim to propose the LBIFS-IBA approach and to study its properties in detail. The proposed approach is a result of the application of an IBA-inspired operation on GIFS with high descriptive power and widespread application possibilities. In this paper, first, we summarize generalizations of IFS definition and provide a brief critical reassessment of these approaches. Furthermore, we aim to introduce LBIFS by generalizing the IFS definition, i.e., by extending the IFS-interpretational triangle into the LBIFS-interpretational square. We also define an IBA-based logical operation for dealing with LBIFS to obtain an approach consistent with Boolean laws and theorems. Finally, we illustrate the applicability of the proposed approach on an example of Japanese candlestick modeling and compare it with traditional IFS and some prominent generalizations. On the one hand, by using LBIFS-interpretational square and IBA-based negation operator, uncertainty interpretation in LBIFS-IBA is enhanced as comparing with existing approaches. Furthermore, there are more options for choosing an appropriate operator due to the IBA framework. On the other hand, IBA-based algebra is computational more expensive and requires deeper knowledge about dependencies in data. Therefore, it may be found as slightly more demanding for application.

This paper is organized as following: The notion of IFS, together with basic mathematical notation and graphical representations are given in Section 2. In Section 3, we recap the finding considering the most prominent generalizations of IFS. The brief overview of IBA is given in Section 4. In Section 5, we introduce IBA-based operations on generalized IFS. Special attention is devoted to uncertainty interpretation in the proposed approach. Furthermore, in Section 6, we illustrate the applicative importance of our approach on several examples. Finally, we present the main conclusions and list potential directions of future work in Section 7.

\section{Intuitionistic Fuzzy Sets}

Intuitionistic fuzzy set theory is one of the most prominent generalizations of the traditional fuzzy set theory [1,2]. IFSs consider both membership and non-membership of an element to a certain set, providing greater description power as compared with traditional fuzzy sets [2]. In the literature, IFS are often called Atanassov IFS due to some terminological issues raised and resolved in the past (for instance, see [32]).

Definition 1 ([1]). An intuitionistic fuzzy set $A$ in a universe $X$ is defined as an object $A=$ $\left\{\left(x, \mu_{A}(x), v_{A}(x) \mid x \in X\right)\right\}=\left\langle\mu_{A}, v_{A}\right\rangle$ wherefunctions $\mu_{A}(x): X \rightarrow[0,1]$ and $\nu_{A}(x): X \rightarrow[0,1]$ represent degrees of membership and non-membership of the element $x$ to the IFS $A$, and for every $x \in X$ the sum of degrees of membership and non-membership is $0 \leq \mu_{A}+v_{A} \leq 1$.

The IFS definition implicitly comprises the existence of a certain level of uncertainty (the degree of non-determinacy) $\pi_{A}(x)$ for every $x \in X$ to IFS $A$. The uncertainty degree is determined by degrees of membership and non-membership:

$$
\pi_{A}=1-\left(\mu_{A}+v_{A}\right)
$$


There are several graphical realizations of IFS. The most common interpretation $[2,33]$ is given in Figure 1a. The values of both membership (thicker line) and non-membership (thinner line) degrees are measured on the same vertical axis.

1

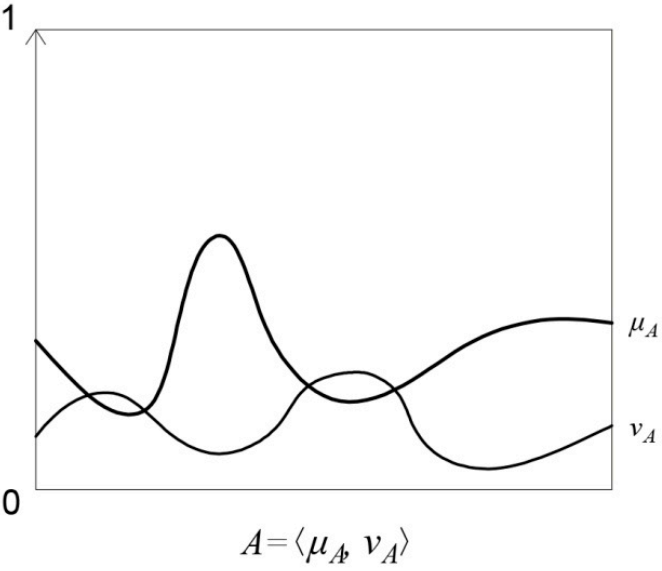

(a)

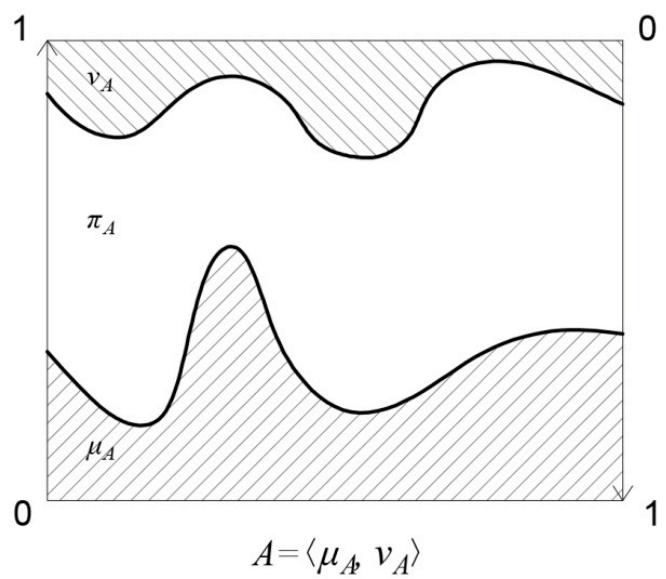

(b)

Figure 1. Graphical representation of an arbitrary IFS: (a) Standard geometrical interpretation of the IFSs; (b) geometrical interpretation with invert axes in order to emphasize uncertainty degree.

Although this is the most widely accepted IFS graphical interpretation, it does not show values of uncertainty degree unambiguously. Therefore, the other well-known graphical interpretation proposed by Atanassov [2,33] depicts degrees of membership and negation of non-membership, together with the degree of uncertainty between them. This graphical interpretation of IFS, with slight modifications in terms of readability by adding additional inverted axis on right side instead of depicting non-membership as $1-v_{A}$ [34], is given in Figure $1 b$.

Finally, any IFS may be graphically illustrated using IFS-interpretational triangle [2], as in Figure 2. The degree of membership to a certain IFS is defined on the horizontal axis, while the non-membership degree is defined on the vertical axis.

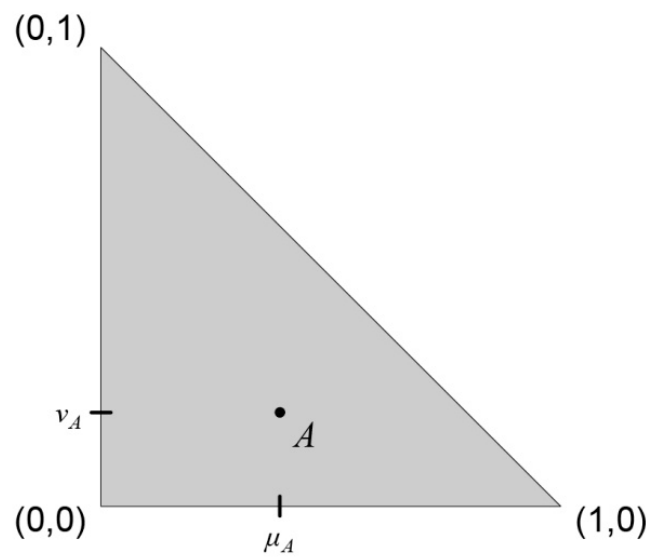

Figure 2. IFS-interpretational triangle.

Several special cases may be distinguished in IFS-interpretational triangle. First, IFS defined in the vertex $(0,0)$ is totally ambiguous, since its degree of non-determinacy is equal to $1\left(\mu_{A}=v_{A}=0, \pi_{A}=1\right)$. Second, IFSs that are on the hypotenuse of IFSinterpretational triangle may be observed as classical fuzzy sets since there is no uncertainty $\left(\mu_{A}+v_{A}=1, \pi_{A}=0\right)$ in these cases. Finally, vertices $(0,1)$ and $(1,0)$ represent special cases of IFSs that are reduced to classical crisp sets, i.e., $\mu_{A}=1, v_{A}=\pi_{A}=0$ or $v_{A}=1, \mu_{A}=$ $\pi_{A}=0$. 
The basic logical operations over IFSs are defined as a natural extension of operations over classical fuzzy sets [1]:

$$
\begin{gathered}
A \wedge B=\left\langle\min \left(\mu_{A}, \mu_{B}\right), \max \left(v_{A}, v_{B}\right)\right\rangle, \\
A \vee B=\left\langle\max \left(\mu_{A}, \mu_{B}\right), \min \left(v_{A}, v_{B}\right)\right\rangle, \\
\neg A=\left\langle v_{A}, \mu_{A}\right\rangle .
\end{gathered}
$$

However, there is a vast corpus of research literature dealing with various extensions and generalizations of basic operations as well as additional IF operators, for example, [2,5,35-37]. Operators of negation and implication are considered to be the most studied ones.

\section{Generalizations of IFS}

One of the main issues in IFS theory concerns the possibility of extending the definition of IFS in terms of the IFS-interpretational triangle. There have been numerous attempts to extend interpretational surface or to establish additional dependence between membership and non-membership degrees.

Herein, we summarize the main finding regarding generalized IFS. In addition, we pay special attention to the definition and interpretation of the uncertainty degree of the particular generalizations. However, we do not reflect on operations on GIFS since they are predominantly direct generalizations of Atanassov's standard IFS operations.

\subsection{Practical Reasons for GIFS}

The need to extend the IFS-interpretational triangle has been identified in the early stages of IFS development by Atanassov [13,33]. Namely, when dealing with practical application, violation of the condition $0 \leq \mu_{A}+v_{A} \leq 1$ is not so rare, i.e., domain experts sometimes neglect this restriction. The reasons for violating IFS restriction ranges from the expert's poor mathematical background, negligence, or even clear intention to describe some phenomenon in this manner.

In determining the degree of membership and degree of non-membership, as well as the degree of uncertainty, we must consider that the same proportion between degrees does not lead to the same interpretation. For example, the same proportion between red, green, and blue colors, but different values of it, does not give the same color, i.e., the same quality. This is one of the topics addressed in [38].

A common approach for dealing with the problem of violation of the IFS condition is the usage of continuous bijective transformations for mapping values from the unit square to the IFS-interpretational triangle [2]. The proposed mappings can be linear or nonlinear functions. Therefore, many generalizations or extensions may be found as redundant or superficial [29]. However, there may be a situation when experts in their respective fields have significant reasons why they assessed values of attributes to violate $\mu_{A}+v_{A} \leq 1$. In these situations, assessed values bear complete knowledge, which may be altered or even lost in the process of transformation, for example, uncertainty degree interpretation. In other words, sometimes it is justified and handy to observe membership and nonmembership independently and go beyond IFS framework. Numerous generalizations and their application in practice speak in favor of that premise. Finally, different generalizations of IFS may be perceived as a step forward in interpreting and comprehending uncertainty in the data.

\subsection{Alternations of IFS Definition}

In [39], six different alternations/generalizations of the IFS definition are proposed along with their graphical interpretation. The idea of alternating IFS definition arose since IFS constraint does not seems to be justified in some practical situations and it could be replaced by some other relations [39]. In this paper, the proposed alternations/generalizations are referred to as generalized Atanassov's intuitionistic fuzzy sets (GAIFS1 to GAIFS6). The definition of one of the presented IFS alternations, GAIFS5, is given below and illustrated in Figure 3. 


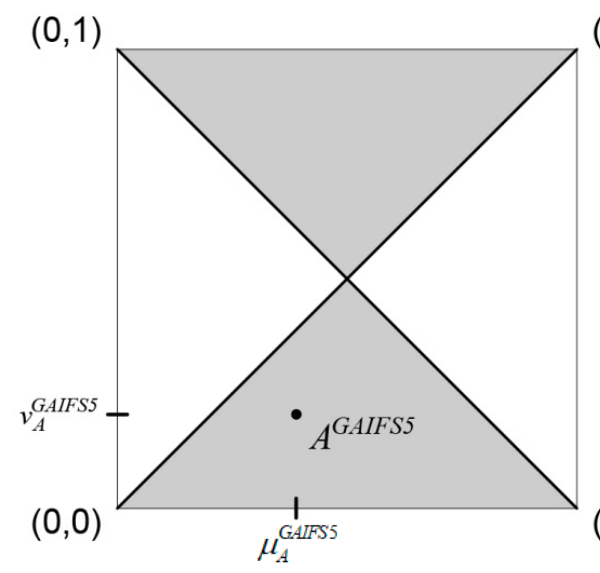

Figure 3. Interpretational surface of generalized IFS in the sense of GAIFS5.

Definition 2 ([39]). A generalized intuitionistic fuzzy set in the sense of GAIFS5 $A^{\text {GAIFS5 }}$ in a universe $X$ is defined as an object $A^{\text {GAIFS5 }}=\left\{\left(x, \mu_{A}^{\text {GAIFS5 }}(x), v_{A}^{\text {GAIFS5 }}(x) \mid x \in X\right)\right\}=$ $\left\langle\mu_{A}^{G A I F S 5}, v_{A}^{G A I F S 5}\right\rangle$ where functions $\mu_{A}^{G A I F S 5}(x), v_{A}^{G A I F S 5}(x): X \rightarrow[0,1]$ and $\left(\mu_{A}^{\operatorname{GAIFS5}}(x) \leq v_{A}^{\operatorname{GAIFS5}}(x) \wedge \mu_{A}^{\operatorname{GAIFS5}}(x)+v_{A}^{\operatorname{GAIFS5}}(x) \geq 1\right) \vee$

$\vee\left(\mu_{A}^{G A I F S 5}(x) \geq v_{A}^{G A I F S 5}(x) \wedge \mu_{A}^{G A I F S 5}(x)+v_{A}^{G A I F S 5}(x) \leq 1\right)$.

The calculation of GAIFS uncertainty degree depends on the GAIFS type. In the general case, values of uncertainty degrees are in the unit interval.

In $[25,29]$, the authors showed that GAIFS1 to GAIFS5 are "in fact intuitionistic fuzzy sets in disguise". In other words, GAIFS1 to GAIFS5, although they can model various problems, might be transformed to IFS with appropriate substitutions of the membership and non-membership functions. That is achievable since the areas of GAIFS1 to GAIFS5 interpretational surfaces are equal to IFS-interpretational triangle. Finally, graphical interpretation of GAIFS6 given in [39] is not correct. It is noticed and elaborated for instance in $[25,29,30]$.

Finally, it should be noted that GAIFS6 coincides with IFS of the second type and that they are further investigated in Section 3.4.

\subsection{Logic-Based IFS Generalization}

In one of the earliest attempts of IFS generalization, Mondal and Samanta suggested extending IFS-interpretational triangle by including logical dependence between membership and non-membership degree as the restriction [16]. In GIFS-MS, the conjunction of membership and non-membership should be less or equal to 0.5 .

Definition 3 ([16]). A generalized intuitionistic fuzzy set in the sense of Mondal and Samanta $A^{M S}$ in a universe $X$ is defined as an object $A^{M S}=\left\{\left(x, \mu_{A}^{M S}(x), v_{A}^{M S}(x) \mid x \in X\right)\right\}=\left\langle\mu_{A}^{M S}, v_{A}^{M S}\right\rangle$ where $\mu_{A}^{M S}(x), v_{A}^{M S}(x): X \rightarrow[0,1]$ and $0 \leq \mu_{A}^{M S} \wedge v_{A}^{M S} \leq 0.5$.

Graphical interpretation of this generalization is given in Figure 4. It is obvious that $B^{M S}=\left\langle\mu_{B}^{M S}, v_{B}^{M S}\right\rangle=\langle 0.2,0.9\rangle$ is valid GIFS-MS, while it is not IFS.

The practical justification of GIFS-MS is that it is not meaningful that degrees of membership and non-membership are significant for certain GIFS simultaneously, i.e., the value of only one degree may exceed 0.5 . Therefore, the minimum operator appears to be a natural choice as a $t$-norm, and it is thoroughly studied [16]. Furthermore, GIFS-MSs are applied for fuzzy filtering [40], multiple criteria decision-making [41], and similarity measuring and pattern recognition [21]. 


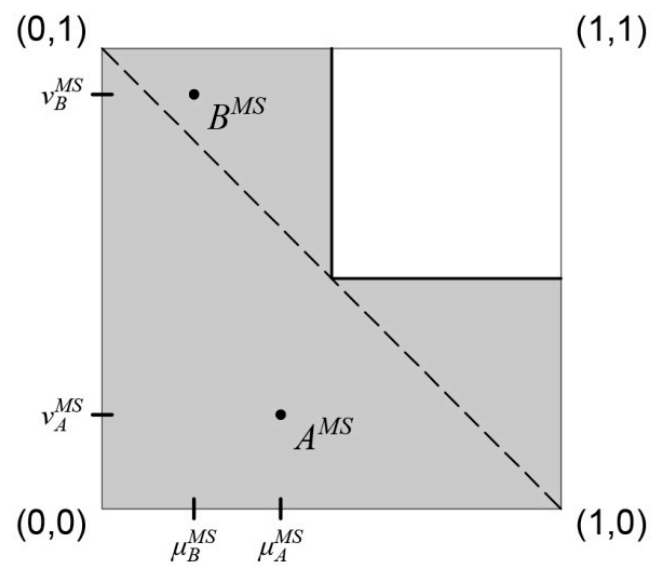

Figure 4. Interpretational surface of GIFS-MS.

The generalized IF index represents the degree of uncertainty of GIFS-MS, and it is given as follows:

$$
\pi_{A}^{M S}=1-\mu_{A}^{M S}-v_{A}^{M S}, \pi_{A}^{M S} \in[-0.5,1]
$$

To the authors' best knowledge, this is the only case in the literature when negative values are allowed for uncertainty degree. Although it may be included in similarity measuring [21], there is no considerable work on the interpretation of GIFS-MS uncertainty degree.

The idea of using any $t$-norm as $\wedge$ operator was analyzed in [42] and led to a more general definition. For instance, the interpretational surface of GIFS-MS when the standard product is used as a $t$-norm is given in Figure 5. Clearly, the interpretational surface is extended. Furthermore, in the case when drastic $t$-norm is utilized, the surface covers almost the whole square, with the exception of the upper and right edge.

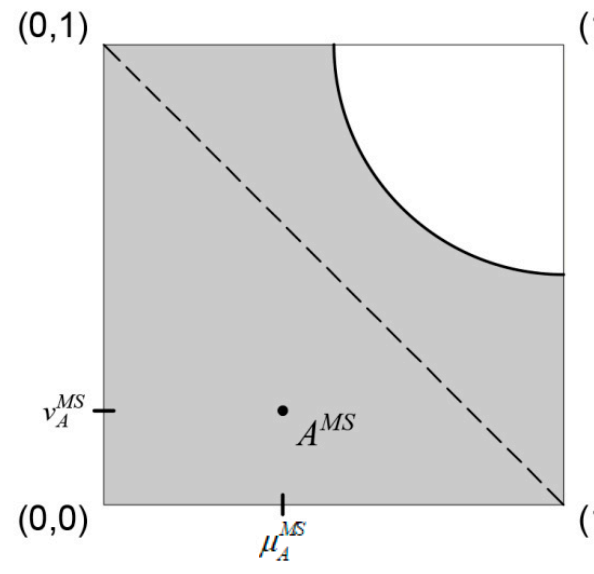

Figure 5. Interpretational surface of GIFS-MS with product as $t$-norm.

\subsection{Power and Root-Type Generalizations of IFS}

The main idea behind power and root-type generalizations of IFS is to raise functions of membership and non-membership to a certain power to increase the interpretational surface of a certain generalized IFS. Raising values to arbitrary power is considered to be the most frequent manner of loosening IFS condition. Thus, power and root-type generalizations of IFS are the most prominent GIFS. However, numerous power-type generalizations of IFS that have been proposed recently have created a type of terminological confusion [24].

The very first attempt of generalizing IFS by squaring membership and non-membership function was named IFS of the second type (IFS-T2) [13]. 
Definition 4 ([13,33]). An intuitionistic fuzzy set of the second type $A^{T 2}$ in a universe $X$ is defined as an object $A^{T 2}=\left\{\left(x, \mu_{A}^{T 2}(x), v_{A}^{T 2}(x) \mid x \in X\right)\right\}=\left\langle\mu_{A}^{T 2}, v_{A}^{T 2}\right\rangle$ where $\mu_{A}^{T 2}(x), v_{A}^{T 2}(x): X \rightarrow[0,1]$ and $0 \leq\left(\mu_{A}^{T 2}\right)^{2}+\left(v_{A}^{T 2}\right)^{2} \leq 1$

The definition of IFS-T2 coincides with Yager's Pythagorean sets [15] and GAIFS6 proposed in [39]. The interpretational surface of these sets is given in Figure 6. Furthermore, these GIFS may be seen as a special case of all parameterized power-type generalizations $[24,43,44]$. Finally, these GIFS, mostly under the term Pythagorean sets, are applied for dealing with a wide range of real-life problems (for instance, see $[22,23,45]$ ).

$(0,1)$

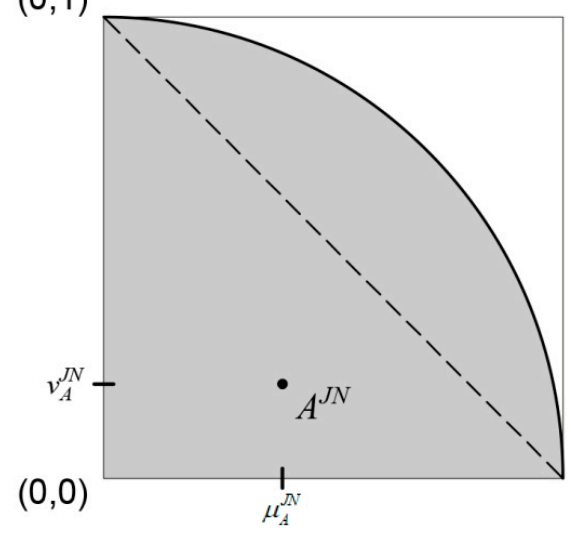

Figure 6. Interpretational surface of IFS-T2/Pythagorean sets/GAIFS6/GIFS-JN for $\delta=2 / \mathrm{q}-\mathrm{ROFS}$ for $q=2 /$ IFS-Tn for $n=2$.

The uncertainty degree of these GIFS in IFS-T2 notation is given as following:

$$
\pi_{A}^{T 2}=\sqrt{1-\left(\mu_{A}^{T 2}\right)^{2}-\left(v_{A}^{T 2}\right)^{2}}, \pi_{A}^{T 2} \in[0,1]
$$

In this manner, the conventional IFS principle that the sum of membership, nonmembership, and uncertainty degrees is equal to 1 is abandoned, since the sum of squares of these functions is equal to 1 . This may inflict the interpretability of IFS-T2, although it increased modeling possibilities.

Analogous to IFS-2T, IFS of the third type has been proposed by cubing membership and non-membership functions [46]. The most general approaches to power-type GIFS involve raising functions of membership and non-membership to a power defined as a parameter, for example, GIFS in the sense of Jamkhaneh and Nadarajah (GIFS-JN) defined in [43] and presented in Figure 6.

Definition 5 ([44]). A generalized intuitionistic fuzzy set in the sense of Jamkhaneh and Nadarajah $A^{J N}$ in a universe $X$ is defined as an object $A^{J N}=\left\{\left(x, \mu_{A}^{J N}(x), v_{A}^{J N}(x) \mid x \in X\right)\right\}=$ $\left\langle\mu_{A}^{J N}, v_{A}^{J N}\right\rangle$ where $\mu_{A}^{J N}(x), v_{A}^{J N}(x): X \rightarrow[0,1]$ and $0 \leq\left(\mu_{A}^{J N}\right)^{\delta}+\left(v_{A}^{J N}\right)^{\delta} \leq 1, \delta=n \vee \delta=$ $1 / n, n \in \mathrm{N}$.

The definition of IFS-JN is very similar to Yager's $q$ th rung orthopair fuzzy sets (qROFS) [44] and Atanassov's IFS of $n$th type (IFS-Tn) [24]. In fact, the only difference is in the range of the parameters, i.e., parameter $q$ in q-ROFS is $q \geq 1$, while $n$ in IFSTn is $n \in \mathrm{R}, n>0$. It should be pointed out that, although interpretational surface of GIFS-JN/q-ROFS/IFS-Tn has no closed form, for $\delta, q, n \rightarrow \infty$ it tends to be equal to the unit square. 
The uncertainty degree of these parameterized GIFS in GIFS-JN notation is given as following:

$$
\pi_{A}^{J N}=\sqrt[\delta]{1-\left(\mu_{A}^{J N}\right)^{\delta}-\left(v_{A}^{J N}\right)^{\delta}}, \pi_{A}^{J N} \in[0,1]
$$

This relation is the direct generalization of IFS-T2 uncertainty degree, featuring the same benefits, as well as shortcomings.

Although IFSs of root type (IFS-RT) [14] share a background with the aforementioned power-type GIFS, they utilize different restrictions.

Definition 6 ([14]). An intuitionistic fuzzy set of root-type $A^{R T}$ in a universe $X$ is defined as an object $A^{R T}=\left\{\left(x, \mu_{A}^{R T}(x), v_{A}^{R T}(x) \mid x \in X\right)\right\}=\left\langle\mu_{A}^{R T}, v_{A}^{R T}\right\rangle$ where $\mu_{A}^{R T}(x), v_{A}^{R T}(x): X \rightarrow[0,1]$ and $0 \leq \sqrt{\left(\mu_{A}^{R T}\right)} / 2+\sqrt{\left(v_{A}^{R T}\right)} / 2 \leq 1$.

The IFS-JN condition $0 \leq \sqrt{\left(\mu_{A}^{R T}\right)} / 2+\sqrt{\left(v_{A}^{R T}\right)} / 2 \leq 1$ is satisfied in the general case since $\mu_{A}^{R T}(x), v_{A}^{R T}(x): X \rightarrow[0,1]$, i.e., the condition is redundant. Thus, the interpretational surface of GIFS-RT is equal to the whole unit square, since the condition is unneeded.

The uncertainty degree of IFS-RT is defined in the same manner as in IFS-T2, and it is given as following:

$$
\pi_{A}^{R T}=\left(1-\sqrt{\mu_{A}^{R T}}-\sqrt{v_{A}^{R T}}\right)^{2}, \pi_{A}^{R T} \in[0,1]
$$

In the case of IFS-RT, the sum of roots of membership, non-membership, and uncertainty degrees is equal to 1 . As in the previously explained generalizations, the sum of membership, non-membership, and uncertainly is not equal to 1 . This is the only GIFS that restriction in its definition is not in relation to the value of uncertainty degree.

\subsection{Generalizations of IFS by Linear Extensions of IFS-Interpretational Triangle}

The main idea behind Liu's generalized IFS (GIFS-L) is to extend IFS-interpretational surface linearly by introducing extensional index $L$ [17]. Parameter $L$ takes values from the real unit interval, and it is added to the upper boundary. Thus, GIFS-L parameterizes IFS definition differently from other GIFS realizations.

Definition 7 ([17]). A Liu's generalized intuitionistic fuzzy set $A^{L}$ in a universe $X$ is defined as an object $A^{L}=\left\{\left(x, \mu_{A}^{L}(x), v_{A}^{L}(x) \mid x \in X\right)\right\}=\left\langle\mu_{A}^{L}, v_{A}^{L}\right\rangle$ where $\mu_{A}^{L}(x), v_{A}^{L}(x): X \rightarrow[0,1]$ and $0 \leq \mu_{A}^{L}+v_{A}^{L} \leq 1+L, L \in[0,1]$.

The example of Liu's generalized IFS-interpretational surface for $L=0.6$ is presented in Figure 7. Liu's generalized IFS offers the greatest descriptive power for $L=1$, transforming GFIS-L-interpretational surface to the unit square.

The generalized intuitionistic fuzzy index of GIFS-L, representing the uncertainty degree, is defined as following:

$$
\pi_{A}^{L}=1+L-\mu_{A}^{L}-v_{A}^{L}, \pi_{A}^{L} \in[0,1+L]
$$

In the most general case for $L=1$, the GIF index is on [0,2] interval. This is the only generalization of IFS that allows values of uncertainty degree greater than 1 . However, the authors did not pay much attention to uncertainty interpretation. Still, this GIFS proved their applicative value on problems of multiple criteria decision-making via Choquet integral [20] and IFS clustering [47]. 


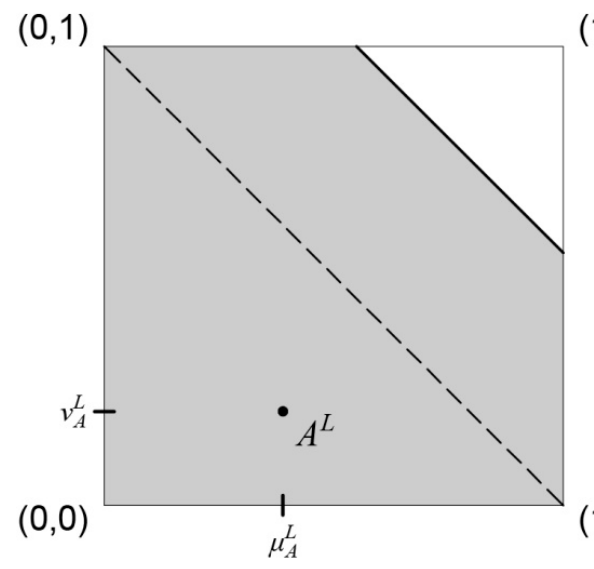

Figure 7. Interpretational surface of GIFS-L for $L=0.6$.

\subsection{Three-Dimensional Generalizations of IFS}

Neutrosophic sets (NSs) [18] are presented as a natural generalization of IFS and paraconsistent sets, underlying the difference between NS and IFS. An NS consists of 3 neutrosophic components representing truth, falsity, and indeterminacy, analogous with membership, non-membership, and uncertainty degrees. However, the values of truth, falsity, and indeterminacy may be assigned independently from each other without any restrictions, unlike in IFS and presented GIFS.

Definition 8 ([18]). A neutrosophic set $A^{N S}$ defined as an object $A^{N S}=\left\langle T_{A}, F_{A}, I_{A}\right\rangle$ where functions $\left.T_{A}, F_{A}, I_{A} \in\right]^{-} 0,1^{+}[$are neutrosophic components.

Since the only limitation for values of neutrosophic components is to be within the unit interval, it follows that:

$$
0 \leq T_{A}+F_{A}+I_{A} \leq 3 .
$$

Therefore, any NS may be presented in 3-dimensional space that is equal to the unit cube.

Spherical fuzzy sets (SFSs) [19], a subset of Smarandache's NS, are also defined in 3-dimensional space. SFS are partly inspired by a spherical representation of IFS [48]. In the case of SFS, values of membership, non-membership, and uncertainty degrees may be defined independently on the unit interval, with the restriction that their squared sum must be on the unit interval. Graphical interpretations of NS and SFS are presented in Figure 8 .

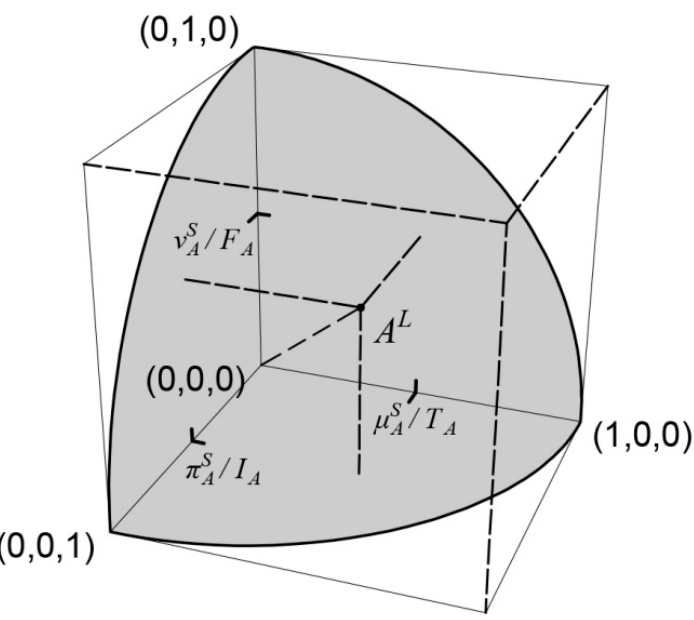

Figure 8. Interpretational cube of NS and interpretational space of SFS. 
Definition 9 ([19]). A spherical fuzzy set $A^{S}$ in a universe $X$ is defined as an object $A^{S}=$ $\left\{\left(x, \mu_{A}^{S}(x), v_{A}^{S}(x), \pi_{A}^{S}(x) \mid x \in X\right)\right\}=\left\langle\mu_{A}^{S}, v_{A}^{S}, \pi_{A}^{S}\right\rangle$ where $\mu_{A}^{S}(x), v_{A}^{S}(x), \pi_{A}^{S}(x): X \rightarrow[0,1]$ and $0 \leq\left(\mu_{A}^{S}\right)^{2}+\left(v_{A}^{S}\right)^{2}+\left(\pi_{A}^{S}\right)^{2} \leq 1$.

Graphical interpretations of NS and SFS are presented in Figure 8.

\subsection{Hybridizations of IFS with Different Types of Sets}

Hybridizations of IFS with some other types of (fuzzy) sets are very popular and a promising direction of IFS theory development [29]. Various approaches to IFS hybridizations are particularly valuable from the applicative point of view. However, these hybridizations are not the primary focus of this paper, therefore, they are only briefly mentioned. Some well-known IFS extensions are interval-valued IFS [13], intuitionistic fuzzy soft sets [49], intuitionistic fuzzy rough sets [50], intuitionistic hesitant fuzzy sets (often referred to as dual hesitant fuzzy sets) [51], complex intuitionistic fuzzy set [52], etc.

\section{Interpolative Boolean Algebra}

Interpolative Boolean algebra is a [0,1]-valued generalization of classical Boolean algebra [31]. IBA is Boolean consistent in the sense that all Boolean axioms and laws are preserved in real-valued case. IBA may be seen as a general framework since it could be utilized as algebra for different types of (fuzzy) sets. In fact, it is used as a foundation for Boolean consistent fuzzy logic [53], the IFS-IBA approach, i.e., an approach to IFS consistent from the intuitionistic point of view [7].

Desired mathematical properties of IBA-based approaches, which are particularly important for various applications, are obtained by distinguishing the symbolic and valued level in IBA. In other words, IBA focuses on the structure of an element instead of the values as in conventional multi-valued (MV) algebras [10]. On the symbolic level, negation is observed and modeled in a different manner as comparing with traditional approaches, which leads to many possible conveniences in applications.

First of all, each logical function should be transformed to a generalized Boolean polynomial (GBP) on the symbolic level of IBA [31].

$$
\varphi \rightarrow \varphi^{\otimes}, \varphi\left(x_{1}, \ldots, x_{n}\right)=\varphi^{\otimes}\left(x_{1}, \ldots, x_{n}\right)
$$

GPSs are polynomials that consist of primary attributes of BA $p_{1}, \ldots, p_{n} \in \Omega$, and generalized product $(\otimes)$, standard plus, and minus as operators. Complex logical expressions $F\left(p_{1}, \ldots, p_{n}\right), G\left(p_{1}, \ldots, p_{n}\right) \in B A(\Omega)$ are mapped to GBP in the following manner:

$$
\begin{aligned}
& (F \wedge G)^{\otimes}=F^{\otimes} \otimes G^{\otimes}, \\
& (F \vee G)^{\otimes}=F^{\otimes}+G^{\otimes}-F^{\otimes} \otimes G^{\otimes}, \\
& (\neg F)^{\otimes}=1-F^{\otimes} .
\end{aligned}
$$

The rules for transforming primary attributes $p_{1}, \ldots, p_{n} \in \Omega$ are:

$$
\begin{aligned}
& \left(p_{i} \wedge p_{j}\right)^{\otimes}=\left\{\begin{array}{l}
p_{i} \otimes p_{j}, i \neq j \\
p_{i}, i=j
\end{array},\right. \\
& \left(p_{i} \vee p_{j}\right)^{\otimes}=p_{i}+p_{j}-\left(p_{i} \wedge p_{j}\right)^{\otimes}, \\
& \left(\neg p_{i}\right)^{\otimes}=1-p_{i}^{\otimes} .
\end{aligned}
$$

On the symbolic level, the operator of generalized product is commutative, associative, and distributive over addition and subtraction. The idempotency of generalized product, defined in the first line of Equation (11), is a significant property. It should be applied on the symbolic level independently of attribute's value realization.

The values of attributes are introduced on the valued level of IBA, after the transformation procedure has been completed. Then, in more detail, all primary attributes are 
valued in a $[0,1]$ interval, and a suitable operator for a generalized product is selected. The final outcome of the valued level of IBA is a value for a given expression in a [0,1] interval.

The generalized product is a subclass of $t$-norms, since it satisfies all four axioms of t-norms (commutativity, associativity, monotonicity, boundary condition), and the additional non-negativity condition [53]. The possibility of using different realizations of GP significantly increases the descriptive power of the IBA approach. The choice of generalized product operator is based on the nature of the primary attributes and/or correlation between them [54]. Three distinctive cases in particular have been studied in the literature [11]: (a) minimum should be used for attributes of the same/similar nature, (b) product should be applied to primary attributes that are statistically independent (different by nature), and (c) Lukasiewicz $t$-norm should be used for negatively correlated attributes.

By introducing three-valued logic, Lukasiewicz paved the way for multi-valued logic. Godel determined the relationship between multi-valued logic and intuitionistic (propositional) logic [55]. A broad spectrum of MV logics is based on the $t$-norm (precisely because of its association with fuzzy logic) with product logic been the one that has been applied the most [56]. IBA-based algebra also defines relations over a real unit interval. However, contrary to other MV approaches, IBA makes a distinction between the structural level and the value level. On the one hand, by introducing structural transformation, IBA-based algebra is computational more expensive and slightly more demanding for application as compared we conventional MV algebras. On the other hand, IBA preserves all Boolean laws in real-valued case, which may be found as uncommon but particularly useful in some situations.

\section{IBA for Generalized IFS}

For the purpose of this paper, we introduce Liu's bipolar I-fuzzy sets (LBIFS) as the special case of GIFS-L with the greatest descriptive power. The formal definition of LBIFS, along with justification and graphical interpretation, is given below in the text. Furthermore, we insist on the interpretation of the uncertainty degree in the case of LBIFS. Finally, we introduce IBA-based operation on LBIFS and examine the validity of Boolean laws within the approach.

\subsection{Liu's Bipolar I-Fuzzy Sets}

Liu's bipolar I-fuzzy sets are defined as the special case of GIFS-L with the maximal value of extensional index $L=1$. Introducing and naming these generalized intuitionisticinspired fuzzy sets as LBIFS seems to be appropriate since the mathematical background and desired characteristics of the approach. Considering the fact that in the proposed approach we aim to focus on Boolean properties of these generalized intuitionistic-inspired fuzzy sets, it is not appropriate to name the approach "intuitionistic". It should also be underlined that the term "bipolar I-fuzzy set" was one of the terms proposed in [32] as an alternative for IFS during the terminological debate. Therefore, it is adopted in the case of our approach.

Definition 10. Liu's bipolar I-fuzzy set $A^{B}$ in a universe $X$ is defined as an object $A^{B}=$ $\left\{\left(x, \mu_{A}^{B}(x), v_{A}^{B}(x) \mid x \in X\right)\right\}=\left\langle\mu_{A}^{B}, v_{A}^{B}\right\rangle$, where $\mu_{A}^{B}(x), v_{A}^{B}(x): X \rightarrow[0,1]$.

The restriction regarding the sum of membership and non-membership degrees is omitted to achieve the maximal descriptive power. Implicitly, it may be stated that:

$$
0 \leq \mu_{A}^{B}(x)+v_{A}^{B}(x) \leq 2
$$

Thus, LBIFSs can be applied for modeling situations when degrees of membership and non-membership overlap, as presented in Figure 9. In other words, they may be perceived as one of the solutions to problems concerning the correctness of the expert estimations of membership and non-membership degrees in the case of classical IFS [2]. 


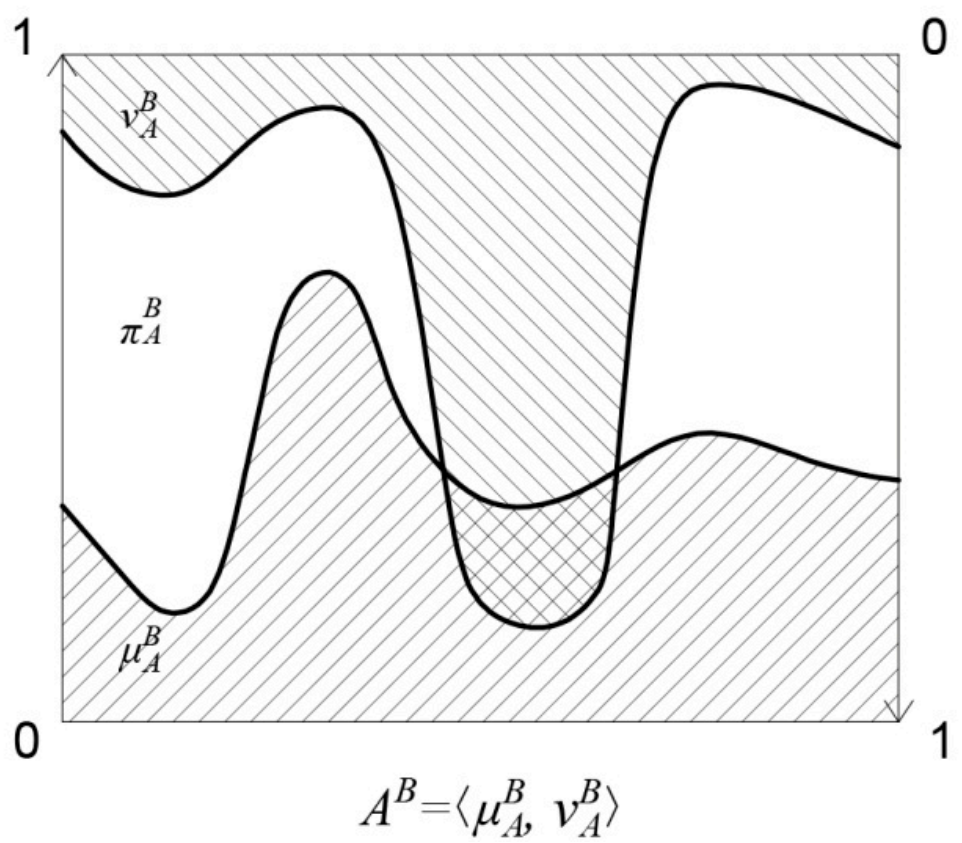

Figure 9. Graphical representation of an arbitrary LBIFS.

In practice, experts commonly violate the definition of IFS, i.e., the sum of membership and non-membership degrees is greater than 1. Conventionally, "incorrect" estimations are transformed into a valid IFS using linear transformations. However, in this case, the meaning of estimations is usually altered significantly. Assuming that values are intentionally defined as such, LBIFS may properly model them by capturing the essence of overlapping estimation.

\subsection{Uncertainty Interpretation and LBIFS-Interpretational Surface}

An interpretational surface of LBIFS is equal to the unit square, as presented in Figure 10. From that point of view, LBIFS may be seen as a generalization of a classic IFS and some of GIFSs, as well as a special case of neutrosophic sets. In addition, the restriction in LBIFS definition coincides with GIFS-RT and GIFS-L for $L=1$. However, they are essentially different from the standpoint of uncertainty degree interpretation.

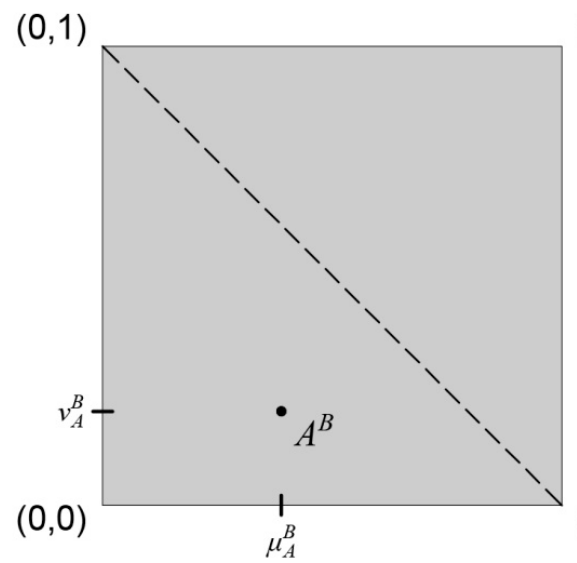

Figure 10. LBIFS-interpretational surface.

The definition of the uncertainty degree of LBIFS is essentially grounded on the uncertainty degree of IFS. It is defined as following:

$$
\pi_{A}^{B}=1-\mu_{A}^{B}-v_{A}^{B} .
$$


However, in the case of LBIFS, the values of uncertainty degree may be negative, i.e., $\pi_{A}^{B}=[-1,1]$. Therefore, the uncertainty degree of LBIFS is described by its intensity as well as its sign. It may be interpreted in a similar manner as the Pearson correlation coefficient. The absolute value of the uncertainty degree depicts level/intensity of uncertainty, while the sign defines its origin (i.e., whether functions of membership and non-membership overlap or not). Thus, we may distinguish three marginal cases:

- If $\pi_{A}^{B}=0$, LBIFS $A$ is absolutely defined, and it is reduced to classical fuzzy set;

- If $\pi_{A}^{B} \in(0,1]$, there is some uncertainty that is defined and interpreted in the same manner as in IFS (uncertainty of non-overlapping) and LBIFS $A$ is reduced to IFS;

- If $\pi_{A}^{B} \in[-1,0)$, there is uncertainty defined by overlapping membership and nonmembership degrees (uncertainty of overlapping). These values of uncertainty also depict a lack of information, but with different origins and interpretation.

For instance, LBIFSs that are in the vertices of LBIFS-interpretational square $(0,0)$ and $(1,1)$ are totally undefined, since the absolute value of uncertainty is equal to 1 in both cases. However, these LBIFS are very different in their meaning since the uncertainty differs in sign. Still, the practical importance of LBIFS is obvious when uncertainty is not high, and it is further elaborated in the next section.

It should be underlined that only GIFS-MS [16], presented in Section 3.3, allows negative values for the uncertainty degree, while for GIFS-L [17] presented in Section 3.5, the length of interval for values of uncertainty may be equal to 2 in a special case. However, neither of those two approaches had provided an explanation of these values for uncertainty degree.

\subsection{IBA-Based Operations on LBIFS}

The logical operations inspired by IBA are introduced in IFS theory in [7], and further investigated in [34]. Thus, they served as a basis for the IBA-IFS approach that is in line with intuitionistic presumptions. However, in the LBIFS-IBA approach, we aim to apply IBA as the foundation of a Boolean consistent approach.

The IBA-based operations of conjunction, disjunction, and negation (intersection, union, and complement in set notation) over LBIFS are defined in the following manner:

$$
\begin{aligned}
& \left(A^{B} \wedge B^{B}\right)^{\otimes}=\left\langle\mu_{A}^{B} \otimes \mu_{B}^{B}, v_{A}^{B}+v_{B}^{B}-v_{A}^{B} \otimes v_{B}^{B}\right\rangle, \\
& \left(A^{B} \vee B^{B}\right)^{\otimes}=\left\langle\mu_{A}^{B}+\mu_{B}^{B}-\mu_{A}^{B} \otimes \mu_{B}^{B}, v_{A}^{B} \otimes v_{B}^{B}\right\rangle, \\
& \left(\neg A^{B}\right)^{\otimes}=\left\langle 1-\mu_{A}^{B}, 1-v_{A}^{B}\right\rangle .
\end{aligned}
$$

Operations of conjunction and disjunction are modeled according to classical IF operations, and realized in the same manner as in the IBA-IFS approach [7,34]. In fact, if the minimum operator is utilized as a generalized product, the LBIFS-IBA conjunction and disjunction come down to standard IF operations.

The negation operator is newly introduced in this paper, and it does not have intuitionistic nature. Instead, it is proposed based on standard negation for classical fuzzy sets. Thus, the usage of this operator is not in line with intuitionistic presumptions, and may be a proper operator for the approach that is Boolean consistent.

Furthermore, the proposed negation operator is not correct in the case of IFS, since in the general case stands:

$$
1-\mu_{A}^{B}+1-v_{A}^{B}=2-\left(\mu_{A}^{B}+v_{A}^{B}\right) \geq 1 \text { if } \mu_{A}^{B}+v_{A}^{B} \leq 1 .
$$

Nevertheless, this negation is valid for LBIFS. It implies that uncertainty degrees of starting LBIFS $A^{B}$ and its negation $\neg A^{B}$ are equal in absolute value, but different in the sign except in the case of a classical fuzzy set:

$$
A^{B}: 1-\mu_{A}^{B}-v_{A}^{B}=\pi_{A}^{B},
$$




$$
\neg A^{B}: 1-\left(1-\mu_{A}^{B}+1-v_{A}^{B}\right)=-\left(1-\mu_{A}^{B}-v_{A}^{B}\right)=-\pi_{A}^{B} .
$$

In the LBIFS-IBA approach, the symbolic and valued levels are distinguished in the same manner as in IBA. Furthermore, the transformation procedure in the case of complex logical expressions is performed analogous to transformation rules given in Equations (10) and (11), and the values and the operator for the generalized product are introduced afterwards. In this way, the mathematical properties of IBA are transferred to the LBIFS-IBA approach.

\subsection{Boolean Properties of the LBIFS-IBA Approach}

The Boolean properties of algebra are the topic that is thoroughly studied in the literature (e.g., see [57]). For instance, there are numerous papers investigating relations between Boolean algebra and fuzzy sets $[6,9,12]$.

Commutativity and distributivity in the LBIFS-IBA approach are directly transferred from IBA and properties of the generalized product. Proofs of these properties are trivial and may be found in [58]. If $0^{B}=\langle 0,1\rangle$ and $1^{B}=\langle 1,0\rangle$ are considered as neutral elements for disjunction and conjunction, identity stands in the general case.

Theorem 1. If $A^{B}=\left\langle\mu_{A}^{B}, v_{A}^{B}\right\rangle$ is a Liu's bipolar I-fuzzy set in a universe $X$, the identity stands in the LBIFS-IBA approach.

Proof of Theorem 1. Since IBA transformation rules and boundary condition of the generalized product, it follows:

$$
\begin{aligned}
\left(A^{B} \vee 0^{B}\right)^{\otimes} & =\left\langle\mu_{A}^{B}, v_{A}^{B}\right\rangle \vee\langle 0,1\rangle= \\
& =\left\langle\mu_{A}^{B}+0-\mu_{A}^{B} \otimes 0, v_{A}^{B} \otimes 1\right\rangle= \\
& =\left\langle\mu_{A}^{B}, v_{A}^{B}\right\rangle= \\
& \equiv A^{B} \\
\left(A^{B} \wedge 1^{B}\right)^{\otimes} & =\left\langle\mu_{A}^{B}, v_{A}^{B}\right\rangle \wedge\langle 1,0\rangle= \\
& =\left\langle\mu_{A}^{B} \otimes 1, v_{A}^{B}+0-v_{A}^{B} \otimes 0\right\rangle= \\
& =\left\langle\mu_{A}^{B}, v_{A}^{B}\right\rangle= \\
& \equiv A^{B}
\end{aligned}
$$

In the theory of intuitionistic fuzzy sets, the validity of the law of excluded middle is of particular interest for many researchers $[2,5,7,34]$. It is commonly studied in four different forms [2]: (1) tautology form, (2) IF tautology form, (3) modified law of excluded middle in tautology form, and (4) modified law of excluded middle in IF tautology form. Since modified forms are not of particular interest when studying Boolean properties and standard tautology form assumes the strongest condition, laws of excluded middle and contradiction are studied in this form.

Theorem 2. If $A^{B}=\left\langle\mu_{A}^{B}, v_{A}^{B}\right\rangle$ is a Liu's bipolar I-fuzzy set in a universe $X$, the law of excluded middle stands in the LBIFS-IBA approach in the tautology form.

Proof of Theorem 2. Since IBA transformation rules and distributivity of generalized product over addition and subtraction, it follows:

$$
\begin{aligned}
\left(A^{B} \vee \neg A^{B}\right)^{\otimes} & =\left\langle\mu_{A}^{B}, v_{A}^{B}\right\rangle \vee\left\langle 1-\mu_{A}^{B}, 1-v_{A}^{B}\right\rangle= \\
& =\left\langle\mu_{A}^{B}+1-\mu_{A}^{B}-\mu_{A}^{B} \otimes\left(1-\mu_{A}^{B}\right), v_{A}^{B} \otimes\left(1-v_{A}^{B}\right)\right\rangle= \\
& =\left\langle\mu_{A}^{B}+1-\mu_{A}^{B}-\mu_{A}^{B}+\mu_{A}^{B} \otimes \mu_{A}^{B}, v_{A}^{B}-v_{A}^{B} \otimes v_{A}^{B}\right\rangle= \\
& =\left\langle 1-\mu_{A}^{B}+\mu_{A}^{B}, v_{A}^{B}-v_{A}^{B}\right\rangle= \\
& \equiv\langle 1,0\rangle
\end{aligned}
$$


Theorem 3. If $A^{B}=\left\langle\mu_{A}^{B}, v_{A}^{B}\right\rangle$ is a Liu's bipolar I-fuzzy set in a universe $X$, the low of contradiction stands in the LBIFS-IBA approach in the tautology form.

Proof of Theorem 3. Since IBA transformation rules and distributivity of generalized product over addition and subtraction, it follows:

$$
\begin{aligned}
\left(A^{B} \wedge \neg A^{B}\right)^{\otimes} & =\left\langle\mu_{A}^{B}, v_{A}^{B}\right\rangle \wedge\left\langle 1-\mu_{A}^{B}, 1-v_{A}^{B}\right\rangle= \\
& =\left\langle\mu_{A}^{B} \otimes\left(1-\mu_{A}^{B}\right), v_{A}^{B}+1-v_{A}^{B}-v_{A}^{B} \otimes\left(1-v_{A}^{B}\right)\right\rangle= \\
& =\left\langle\mu_{A}^{B}-\mu_{A}^{B} \otimes \mu_{A}^{B}, v_{A}^{B}+1-v_{A}^{B}-v_{A}^{B}+v_{A}^{B} \otimes v_{A}^{B}\right\rangle= \\
& =\left\langle\mu_{A}^{B}-\mu_{A}^{B}, 1-v_{A}^{B}+v_{A}^{B}\right\rangle= \\
& \equiv\langle 0,1\rangle
\end{aligned}
$$

As stated previously, the negation operator in the LBIFS-IBA approach is newly introduced in this paper with an idea to mimic classical fuzzy negation. Thus, it is crucial to investigate if it has intuitionistic nature, or is in line with Boolean algebra, i.e., it is involutive.

Theorem 4. If $A^{B}=\left\langle\mu_{A}^{B}, v_{A}^{B}\right\rangle$ is a Liu's bipolar I-fuzzy set in a universe $X$, the double negation rule stands in the LBIFS-IBA approach, i.e., negation is involutive.

Proof of Theorem 4. Since IBA transformation rules, it follows:

$$
\begin{aligned}
\left(\neg\left(\neg A^{B}\right)\right)^{\otimes} & =\neg\left\langle 1-\mu_{A}^{B}, 1-v_{A}^{B}\right\rangle= \\
& =\left\langle 1-\left(1-\mu_{A}^{B}\right), 1-\left(1-v_{A}^{B}\right)\right\rangle= \\
& =\left\langle\mu_{A}^{B}, v_{A}^{B}\right\rangle .
\end{aligned}
$$

Finally, we aim to investigate the validity of De Morgan's laws within the proposed approach.

Theorem 5. If $A^{B}=\left\langle\mu_{A}^{B}, v_{A}^{B}\right\rangle$ and $B^{B}=\left\langle\mu_{B}^{B}, v_{B}^{B}\right\rangle$ are Liu's bipolar I-fuzzy sets in a universe $X$, De Morgan's laws stand in the LBIFS-IBA approach.

Proof of Theorem 5. Since IBA transformation rules, and boundary condition of the generalized product as well as distributivity of generalized product over addition and subtraction, it follows:

$$
\begin{aligned}
& \begin{aligned}
\neg\left(A^{B} \vee B^{B}\right)^{\otimes} & =\neg\left\langle\mu_{A}^{B}+\mu_{B}^{B}-\mu_{A}^{B} \otimes \mu_{B}^{B}, v_{A}^{B} \otimes v_{B}^{B}\right\rangle= \\
& =\left\langle 1-\mu_{A}^{B}-\mu_{B}^{B}+\mu_{A}^{B} \otimes \mu_{B}^{B}, 1-v_{A}^{B} \otimes v_{B}^{B}\right\rangle
\end{aligned} \\
& \begin{aligned}
\left(\neg A^{B} \wedge \neg B^{B}\right)^{\otimes} & =\left\langle 1-\mu_{A}^{B}, 1-v_{A}^{B}\right\rangle \wedge\left\langle 1-\mu_{B}^{B}, 1-v_{B}^{B}\right\rangle= \\
= & \left\langle\left(1-\mu_{A}^{B}\right) \otimes\left(1-\mu_{B}^{B}\right), 1-v_{A}^{B}+1-v_{B}^{B}-\left(1-v_{A}^{B}\right) \otimes\left(1-v_{B}^{B}\right)\right\rangle= \\
= & \left\langle 1-\mu_{A}^{B}-\mu_{B}^{B}+\mu_{A}^{B} \otimes \mu_{B}^{B}, 2-v_{A}^{B}-v_{B}^{B}-1+v_{A}^{B}+v_{B}^{B}-v_{A}^{B} \otimes v_{B}^{B}\right\rangle= \\
= & \left\langle 1-\mu_{A}^{B}-\mu_{B}^{B}+\mu_{A}^{B} \otimes \mu_{B}^{B}, 1-v_{A}^{B} \otimes v_{B}^{B}\right\rangle
\end{aligned} \\
& \text { Thus, } \neg\left(A^{B} \vee B^{B}\right)^{\otimes}=\left(\neg A^{B} \wedge \neg B^{B}\right)^{\otimes} . \\
& \begin{aligned}
\neg\left(A^{B} \wedge B^{B}\right)^{\otimes} & =\neg\left\langle\mu_{A}^{B} \otimes \mu_{B}^{B}, v_{A}^{B}+v_{B}^{B}-v_{A}^{B} \otimes v_{B}^{B}\right\rangle= \\
& =\left\langle 1-\mu_{A}^{B} \otimes \mu_{B}^{B}, 1-v_{A}^{B}-v_{B}^{B}+v_{A}^{B} \otimes v_{B}^{B}\right\rangle
\end{aligned}
\end{aligned}
$$




$$
\begin{aligned}
\left(\neg A^{B} \vee \neg B^{B}\right)^{\otimes} & =\left\langle 1-\mu_{A}^{B}, 1-v_{A}^{B}\right\rangle \vee\left\langle 1-\mu_{B}^{B}, 1-v_{B}^{B}\right\rangle= \\
& =\left\langle 1-\mu_{A}^{B}+1-\mu_{B}^{B}-\left(1-\mu_{A}^{B}\right) \otimes\left(1-\mu_{B}^{B}\right),\left(1-v_{A}^{B}\right) \otimes\left(1-v_{B}^{B}\right)\right\rangle= \\
& =\left\langle 2-\mu_{A}^{B}-\mu_{B}^{B}-1+\mu_{A}^{B}+\mu_{B}^{B}-\mu_{A}^{B} \otimes \mu_{B}^{B}, 1-v_{A}^{B}-v_{B}^{B}+v_{A}^{B} \otimes v_{B}^{B}\right\rangle= \\
& =\left\langle 1-\mu_{A}^{B} \otimes \mu_{B}^{B}, 1-v_{A}^{B}-v_{B}^{B}+v_{A}^{B} \otimes v_{B}^{B}\right\rangle \\
\text { Thus, } \neg\left(A^{B} \wedge\right. & \left.B^{B}\right)^{\otimes}=\left(\neg A^{B} \vee \neg B^{B}\right)^{\otimes} .
\end{aligned}
$$

Therefore, it is concluded that the LBIFS-IBA approach is consistent with Boolean frame.

\section{An Example of the LBIFS-IBA Approach Application to Candlestick Modeling}

The main benefits of the LBIFS-IBA approach as compared with traditional IFS techniques are illustrated on the example of financial technical analysis. More specifically, the proposed approach is applied for price charting analysis using Japanese candlesticks.

Japanese candlestick financial chart analysis is a technique often used to describe and model price movements of stocks, currencies, or derivates [59]. Each candlestick represents price movement during a fixed period of time, for example, one day, one hour, etc. It is characterized by four distinctive prices:

- $\quad$ Open $(\mathrm{O})$, the opening price during the observed time period;

- High $(\mathrm{H})$, representing the highest price during the observed time period;

- Low (L), representing the lowest price during the observed time period;

- $\quad$ Close $(\mathrm{C})$, the closing price during the observed time period.

The candlestick consists of the real body, and the upper and lower shadow. The real body may be white in the case of raising price trend $(\mathrm{O}<\mathrm{C})$, or black in the case of declining price trend $(\mathrm{O}>\mathrm{C})$ during the observed period of time. The length of a real body is equal to the absolute difference of $\mathrm{O}$ and $\mathrm{C}$ prices. Therefore, the shadows are indicating how much the price has fluctuated relative to $\mathrm{O}$ and $\mathrm{C}$ prices. Values of $\mathrm{H}$ and $\mathrm{L}$ are the top and the bottom of the upper and lower shadow.

The size and color of the body and shadows, i.e., price relations and ratios, are used for building expert-based patterns and trading models and strategies [59]. However, these models are often fuzzified in order to improve their descriptive power, for example, [60-62].

\subsection{Presentation of Japanese Candlestick Using the LBIFS-IBA Approach}

The LBIFS-IBA approach may be seen as a useful tool for Japanese candlestick modeling and analysis. Namely, we may transform a sequence of candlesticks in a corresponding LBIFS in two steps:

- Determining the lowest $\mathrm{L}$ and the highest $\mathrm{H}$ of all candlesticks;

- Obtaining degrees of memberships and non-membership for each candlestick via minmax normalization of $\mathrm{O}$ and $\mathrm{C}$ with respect to determined maximum and minimum.

By using the invert procedure, a sequence of candlesticks may be obtained from LBIFS. It should be underlined that this is only one of many possible manners to model Japanese candlesticks using LBIFS or IFS in general.

Thus, let us consider a sequence of three candlesticks presented on the left-hand side of Figure 11. We may present them using the LBIFS-IBA approach as on the right-hand side of Figure 11. The real bodies of candlesticks are presented as surfaces of uncertainty. Evidently, GIFSs are an appropriate tool for modeling this situation because of the possibility of overlapping membership and non-membership functions. Mapping GIFS to IFS does not make much sense in this case, since overlapping has a clear-cut meaning distinct of not-overlapping. More precisely, non-overlapping indicates a rising trend in data, while overlapping indicates a declining trend. In such a way, we retain information and interpretability of data that is particularly important for financial modeling and decisionmaking. This approach is very convenient when we aim to aggregate daily data on the weekly level and still preserve information about some daily tendencies. Furthermore, this may be seen as a significant advantage of the LBIFS-IBA approach as compared with traditional approaches. 


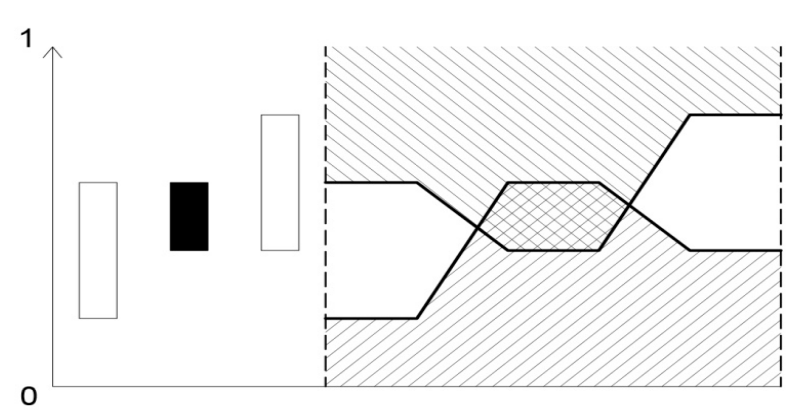

Figure 11. A sequence of candlesticks and the corresponding LBIFS.

\subsection{Uncertainty Interpretation and the Benefits of Negation Operator}

One of the essential differences of the proposed LBIFS-IBA approach as compared with traditional and generalized IFS is the interpretation of uncertainty that may be easily illustrated on the example of negation.

Let us present a single candlestick (Figure 12a) as LBIFS and calculate its negation in LBIFS-IBA and traditional IFS approaches (Figure 12b,c, respectively).

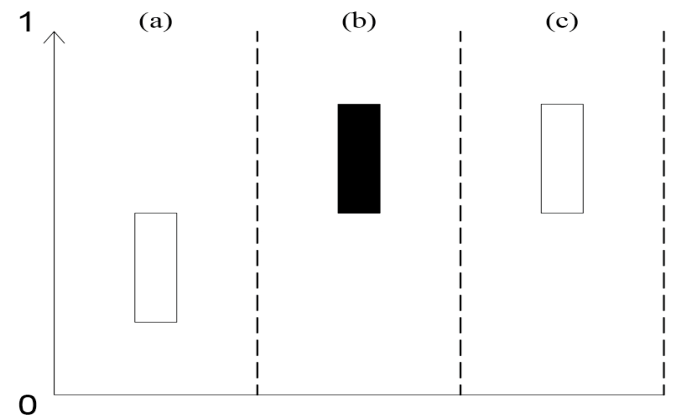

Figure 12. Illustration of negation in the LBIFS-IBA approach on the example of candlestick modeling: (a) a single candlestick; (b) negation of the candlestick given in (a) in LBIFS-IBA approach; (c) negation of the candlestick given in (a) in traditional IFS approach.

It is obvious that the real bodies of all three candlesticks are of the same length, i.e., all three variables are equal in terms of uncertainty intensity:

$$
\begin{aligned}
& A=A^{B}: \pi_{A}=1-\mu_{A}-v_{A}=1-\mu_{A}^{B}-v_{A}^{B}, \\
& \neg A: \pi_{\neg A}=1-\mu_{\neg A}-v_{\neg A}=1-v_{A}-\mu_{A}, \\
& \neg A^{B}:\left|\pi_{\neg A}^{B}\right|=\left|1-\mu_{\neg A}^{B}-v_{\neg A}^{B}\right|=\left|-1+\mu_{A}^{B}+v_{A}^{B}\right|=1-\mu_{A}^{B}-v_{A}^{B} .
\end{aligned}
$$

Furthermore, both candlesticks, which represent the negation of the initial one, are "positioned" in the same manner, although they differ in color. Bearing in mind that the color of a candlestick represents the trend in prices, it seems that LBIFS realization is more meaningful, i.e., negation of raising candlestick is the one that is declining.

Finally, let us consider an example regarding modeling different candlestick using some IFS generalizations. In Figure 13, five candlesticks are presented. The first two candlesticks represent a rising price trend, while the others are declining. It is also important to emphasize that the first three candlesticks are of the same length. 


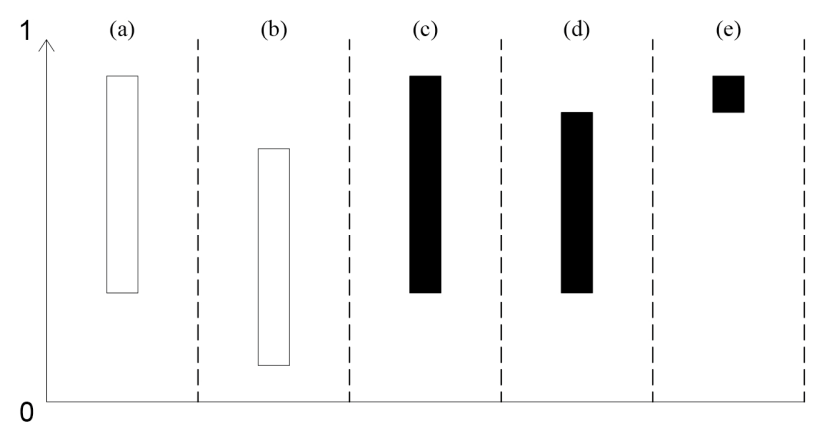

Figure 13. Candlesticks used for comparison of IFS generalizations. (a) $\left\langle\mu_{A}, v_{A}\right\rangle=\langle 0.3,0.1\rangle$ (b) $\left\langle\mu_{A}, v_{A}\right\rangle=\langle 0.1,0.3\rangle$ (c) $\left\langle\mu_{A}, v_{A}\right\rangle=\langle 0.9,0.7\rangle$ (d) $\left\langle\mu_{A}, v_{A}\right\rangle=\langle 0.8,0.7\rangle$ (e) $\left\langle\mu_{A}, v_{A}\right\rangle=\langle 0.9,0.2\rangle$.

In order to analyze and compare various IFS generalizations, we have chosen several of the most used ones. Uncertainty degrees for various IFS generalizations and variants (GIFS-MS, IFS-T2, IFS-Tn for $n=3$, GIFS-L for $L=0.2$, GIFS-L for $L=1$, IFS transformed using bijective mapping as in [2], and in the LBIFS-IBA approach) are calculated and given in Table 1. If IFS cannot be modeled using a certain generalization, uncertainty degree is not calculated and marked with an " $\mathrm{X}$ ".

Table 1. Uncertainty degrees for various IFS generalizations.

\begin{tabular}{|c|c|c|c|c|c|c|c|c|}
\hline \multicolumn{2}{|c|}{ Input } & \multirow{2}{*}{$\pi_{A}^{M S}$} & \multirow{2}{*}{$\pi_{A}^{T 2}$} & \multirow{2}{*}{$\pi_{A}^{T n}, n=3$} & \multirow{2}{*}{$\begin{array}{c}\pi_{A}^{L}, L= \\
0.2\end{array}$} & \multirow{2}{*}{$\pi_{A^{\prime}}^{L} L=1$} & \multirow{2}{*}{$\pi_{A}^{\text {trans }}$} & \multirow{2}{*}{$\pi_{A}^{B}$} \\
\hline$\mu_{A}$ & $v_{A}$ & & & & & & & \\
\hline 0.30 & 0.10 & 0.60 & 0.95 & 0.99 & 0.80 & 1.60 & 0.70 & 0.60 \\
\hline 0.10 & 0.30 & 0.60 & 0.95 & 0.99 & 0.80 & 1.60 & 0.70 & 0.60 \\
\hline 0.90 & 0.70 & $x$ & $x$ & $x$ & $x$ & 0.40 & 0.10 & -0.60 \\
\hline 0.80 & 0.70 & $x$ & $x$ & 0.53 & $x$ & 0.50 & 0.20 & -0.50 \\
\hline 0.90 & 0.20 & -0.10 & 0.39 & 0.64 & 0.10 & 0.90 & 0.10 & -0.10 \\
\hline
\end{tabular}

All approaches are able to model the first two candlesticks. However, uncertainty degrees for IFS-T2 and IFS-Tn for $n=3$, are quite large, i.e., $\pi_{A}^{T 2}$ and $\pi_{A}^{T n}, n=3$ tend to 1 . This hinders the interpretation of these sets. In addition, the uncertainty degree of GIFS-L for $L=1$ is larger than 1 , which is rather unusual and hard to comprehend. Finally, the sum of uncertainty degree for IFS transformed using bijective mapping, and starting values of membership and non-membership is not equal to 1 . However, the degree of uncertainty in our approach is not scaled, and it has a clear-cut meaning and interpretation.

The third candlestick has high values for both membership and non-membership. Therefore, only three of seven approaches can model this candlestick: GIFS-L for $L=1$, IFS transformed using bijective mapping, and in the LBIFS-IBA approach. Although the fourth case is very similar to the third one, IFS-Tn for $n=3$ is able to model it; however, it is still out of range for GIFS-MS, IFS-T2, and GIFS-L for $L=0.2$.

A potential shortcoming of IFS transformed using bijective mapping may occur when dealing with sets with a high degree of membership. Namely, both cases 3 and 5 present candlesticks are with high membership degrees. However, they differ in non-membership degree and length, which is oblivious in Figure 13, and the uncertainty degree of IFS transformed using bijective mapping fails to recognize this.

Finally, we may conclude that all presented candlesticks are successfully modeled using LBIFS-IBA. Uncertainty degrees $\pi_{A}^{B}$ are easy to understand and interpret, and it is particularly suitable that declining candlesticks are with negative uncertainty degrees. 


\section{Conclusions and Directions of Future Research}

The purpose of this paper is to introduce the LBIFS-IBA approach and study its properties. The main idea was to define and apply IBA-based logical operations on the most general two-dimensional GIFS.

In order to build a solid foundation for the research, an extensive overview of GIFS is given, reflecting on the descriptive power and resemblance of many GIFSs. Lius's GIFS with maximal value of parameter $L$ is chosen as the basic one for defining LBIFS. Furthermore, IBA-based operations are introduced for dealing with LBIFS, generating the LBIFS-IBA approach. The mathematical properties of the approach are studied, with a special focus on laws of excluded middle and contradiction. From the standpoint of practical justification, particular attention is committed to uncertainty interpretation within our approach. The uncertainty degree of LBIFS is described by its intensity as well as its sign. The clear distinction is made between the uncertainty of non-overlapping (positive values of uncertainty degree) and uncertainty of overlapping (negative values of uncertainty degree) in terms of interpretation. Finally, the LBIFS-IBA approach is compared to the traditional IFS using the illustrative example regarding candlestick charting modeling and analysis. It is shown that LBIFS-IBA is a superior approach to traditional IFS generalization in terms of description power and uncertainty interpretation.

The main directions of our future work cover both practical and theoretical aspects. We aim to define a framework for similarity modeling in the LBIFS-IBA approach to recognize candlestick patterns as trading signals. We plan to focus on investigating the modus and potential of introducing IBA-inspired operations to three-dimensional generalizations of IFS.

Author Contributions: Conceptualization, P.M. and B.P.; methodology, P.M.; validation, P.M., B.P., and I.D.; formal analysis, P.M. and B.P.; investigation, P.M., B.P., and I.D.; writing-original draft preparation, P.M.; writing-review and editing, B.P. and I.D.; visualization, P.M.; supervision, B.P. All authors have read and agreed to the published version of the manuscript.

Funding: This research received no external funding.

Institutional Review Board Statement: Not applicable.

Informed Consent Statement: Not applicable.

Data Availability Statement: Not applicable.

Acknowledgments: The authors are thankful to the anonymous reviewers for their valuable remarks that improved the readability of the paper.

Conflicts of Interest: The authors declare no conflict of interest.

\section{References}

1. Atanassov, K.T. Intuitionistic fuzzy sets. Fuzzy Sets Syst. 1986, 20, 87-96. [CrossRef]

2. Atanassov, K.T. Intuitionistic Fuzzy Sets-Theory and Applications; Springer: Berlin/Heidelberg, Germany, 2012. [CrossRef]

3. Atanassov, K.T.; Gargov, G. Interval valued intuitionistic fuzzy sets. Fuzzy Sets Syst. 1989, 31, 343-349. [CrossRef]

4. Jun, Y.B.; Kim, K.H. Intuitionistic fuzzy ideals of BCK-algebras. Int. J. Math. Math. Sci. 2000, 24, 839-849. [CrossRef]

5. Bustince, H.; Barrenechea, E.; Pagola, M. Generation of interval-valued fuzzy and Atanassov's intuitionistic fuzzy connectives from fuzzy connectives and from $\mathrm{K} \alpha$ operators: Laws for conjunctions and disjunctions, amplitude. Int. J. Intell. Syst. 2008, 23, 680-714. [CrossRef]

6. Tripathy, B.K.; Satapathy, M.K.; Choudhury, P.K. Intuitionistic fuzzy lattices and intuitionistic fuzzy Boolean algebras. Int. J. Eng. Tech. 2013, 5, 2352-2361.

7. Milošević, P.; Poledica, A.; Rakićević, A.; Petrović, B.; Radojević, D. Introducing interpolative Boolean algebra into intuitionistic fuzzy sets. In Proceedings of the 9th EUSFLAT Conference, Gijón, Spain, 30 June-3 July 2015; Alonso, J.M., Bustince, H., Reformat, M., Eds.; Atlantis Press: Dordrecht, The Netherlands, 2015; pp. 1389-1394. [CrossRef]

8. Xue, Z.; Xiao, Y.; Liu, W.; Cheng, H.; Li, Y. Intuitionistic fuzzy filter theory of BL-algebras. Int. J. Mach. Learn. Cyb. 2013, 4, 659-669. [CrossRef]

9. Radojević, D. Fuzzy set theory in Boolean frame. Int. J. Comput. Commun. Control 2008, 3, 121-131.

10. Trillas, E. Non contradiction, excluded middle, and fuzzy sets. In International Workshop on Fuzzy Logic and Applications; Springer: Berlin/Heidelberg, Germany, 2009; pp. 1-11. [CrossRef] 
11. Dragović, I.; Turajlić, N.; Radojević, D.; Petrović, B. Combining Boolean consistent fuzzy logic and AHP illustrated on the web service selection problem. Int. J. Comput. Intell. Syst. 2014, 7, 84-93. [CrossRef]

12. Coulon, J.; Coulon, J.L. Fuzzy Boolean algebras. J. Math. Anal. Appl. 1984, 99, 248-256. [CrossRef]

13. Atanassov, K.T. Intuitionistic Fuzzy Sets; Springer: Berlin/Heidelberg, Germany, 1999. [CrossRef]

14. Srinivasan, R.; Palaniappan, N. Some operators on intuitionistic fuzzy sets of root type. Ann. Fuzzy Math. Inform. 2012, 4, 377-383.

15. Yager, R.R. Pythagorean membership grades in multicriteria decision making. IEEE Trans. Fuzzy Syst. 2013, $22,958-965$. [CrossRef]

16. Mondal, T.K.; Samanta, S.K. Generalized intuitionistic fuzzy sets. J. Fuzzy Math. 2002, 10, 839-862.

17. Liu, H.C. Liu's generalized intuitionistic fuzzy sets. J. Educ. Meas. Stat. 2010, 18, 1-14.

18. Smarandache, F. Neutrosophic set-a generalization of the intuitionistic fuzzy set. Int. J. Pure Appl. Math. 2005, 24, $287-297$.

19. Kutlu Gündoğdu, F.; Kahraman, C. Spherical fuzzy sets and spherical fuzzy TOPSIS method. J. Intell. Fuzzy Syst. 2019, 36, 337-352. [CrossRef]

20. Wu, J.; Chen, F.; Nie, C.; Zhang, Q. Intuitionistic fuzzy-valued Choquet integral and its application in multicriteria decision making. Inform. Sci. 2013, 222, 509-527. [CrossRef]

21. Park, J.H.; Hwang, J.; Kim, J.; Park, B.; Park, J.; Son, J.; Lee, S. Similarity measure between generalized intuitionistic fuzzy sets and its application to pattern recognition. J. Comput. Anal. Appl. 2016, 20, 984-994.

22. Ak, M.F.; Gul, M. AHP-TOPSIS integration extended with Pythagorean fuzzy sets for information security risk analysis. Complex Intell. Syst. 2019, 5, 113-126. [CrossRef]

23. Chen, T.Y. New Chebyshev distance measures for Pythagorean fuzzy sets with applications to multiple criteria decision analysis using an extended ELECTRE approach. Expert Syst. Appl. 2020, 147, 113-164. [CrossRef]

24. Atanassov, K.T.; Vassilev, P. On the intuitionistic fuzzy sets of n-th type. In Advances in Data Analysis with Computational Intelligence Methods; Gaweda, A.E., Kacprzyk, J., Rutkowski, L., Yen, G.G., Eds.; Springer: Cham, Switzerland, 2018; pp. 265-274. [CrossRef]

25. Vassilev, P.; Atanassov, K.T. Generalised Atanassov Intuitionistic Fuzzy Sets Are Actually Intuitionistic Fuzzy Sets. In Intuitionistic and Type-2 Fuzzy Logic Enhancements in Neural and Optimization Algorithms: Theory and Applications; Castillo, O., Melin, P., Kacprzyk, J., Eds.; Springer: Cham, Switzerland, 2020; pp. 107-117. [CrossRef]

26. Atanassov, K.T.; Vassilev, P. Intuitionistic fuzzy sets and other fuzzy sets extensions representable by them. J. Intell. Fuzzy Syst. 2020, 38, 525-530. [CrossRef]

27. Smarandache, F. Neutrosophic set is a generalization of intuitionistic fuzzy set, inconsistent intuitionistic fuzzy set (picture fuzzy set, ternary fuzzy set), pythagorean fuzzy set, spherical fuzzy set, and q-rung orthopair fuzzy set, while neutrosophication is a generalization of regret theory, grey system theory, and three-ways decision (revisited). J. New Theory 2019, 29, 1-31.

28. Vassilev, P.; Atanassov, K.T. A Note on Intuitionistic Fuzzy Sets, Interval Valued Intuitionistic Fuzzy Sets and Picture Fuzzy Sets. In International Workshop on Intuitionistic Fuzzy Sets and Generalized Nets; Springer: Cham, Switzerland, 2018; pp. 24-28. [CrossRef]

29. Vassilev, P.; Atanassov, K.T. Extensions and Modifications of Intuitionistic Fuzzy Sets; "Prof. Marin Drinov" Academic Publishing House: Sofia, Bulgaria, 2019.

30. Milošević, P.; Petrović, B. Interpolative Boolean Algebra for Generalizations of Intuitionistic Fuzzy Sets. In Proceedings of the 2019 Conference of the International Fuzzy Systems Association and the European Society for Fuzzy Logic and Technology, Prague, Czech Republic, 9-13 September 2019; Novák, V., Mařík, V., Štěpnička, M., Navara, M., Hurtík, P., Eds.; Atlantis Press: Dordrecht, The Netherlands, 2019; pp. 676-681. [CrossRef]

31. Radojević, D. [0, 1]-valued logic: A natural generalization of Boolean logic. Yugoslav J. Oper. Res. 2000, 10, $185-216$.

32. Dubois, D.; Gottwald, S.; Hajek, P.; Kacprzyk, J.; Prade, H. Terminological difficulties in fuzzy set theory-The case of "Intuitionistic Fuzzy Sets". Fuzzy Set. Syst. 2005, 156, 485-491. [CrossRef]

33. Atanassov, K.T. Geometrical interpretation of the elements of the intuitionistic fuzzy objects. Int. J. Bioautom. 2016, 20, S27-S42.

34. Milošević, P.; Petrović, B.; Jeremić, V. IFS-IBA similarity measure in machine learning algorithms. Expert Syst. Appl. 2017, 89, 296-305. [CrossRef]

35. Chen, T.Y. Remarks on the Subtraction and Division Operations over Intuitionistic Fuzzy Sets and Interval-Valued Fuzzy Sets. Int. J. Fuzzy Syst. 2007, 9, 169-172. [CrossRef]

36. Atanassov, K.T. Third Zadeh's intuitionistic fuzzy implication. Mathematics 2021, 9, 619. [CrossRef]

37. Atanassov, K.T. Type-1 fuzzy sets and intuitionistic fuzzy sets. Algorithms 2017, 10, 106. [CrossRef]

38. Cuong, B.C.; Kreinovich, V. Picture Fuzzy Sets-a new concept for computational intelligence problems. In Proceedings of the 2013 Third World Congress on Information and Communication Technologies (WICT 2013), Hanoi, Vietnam, 15-18 December 2013; IEEE: Piscataway, NJ, USA, 2013; pp. 1-6. [CrossRef]

39. Despi, I.; Opris, D.; Yalcin, E. Generalised Atanassov Intuitionistic Fuzzy Sets. In Proceedings of the Fifth International Conference on Information, Process, and Knowledge Management 2013 (eKNOW 2013), Nice, France, 24 February-1 March 2013; Malzahn, D., Ed.; IARIA: Wilmington, DE, USA, 2013; pp. 51-56.

40. Park, J.H.; Park, J.K. Hausdorffness on generalized intuitionistic fuzzy filters. Inform. Sci. 2004, 168, 95-110. [CrossRef]

41. Adak, K.; Manna, D.; Bhowmik, M.; Pal, M. TOPSIS in Generalized Intuitionistic Fuzzy Environment. In Handbook of Research on Modern Optimization Algorithms and Applications in Engineering and Economics; Vasant, P., Weber, G.W., Dieu, V.N., Eds.; IGI Global: Hershey, PA, USA, 2016; pp. 630-642. [CrossRef] 
42. Samanta, A.; Samanta, S.K. ( $\left.\mathrm{t}-\mathrm{t}^{*}\right)$-Intuitionistic fuzzy sets. In Proceedings of the 3rd International IEEE Conference Intelligent Systems, London, UK, 4-6 September 2006; IEEE: Piscataway, NJ, USA, 2006; Volume 1482, pp. 771-775. [CrossRef]

43. Jamkhaneh, E.B.; Nadarajah, S. A new generalized intuitionistic fuzzy sets. Hacet. J. Math. Stat. 2015, 44, 1537-1551. [CrossRef]

44. Yager, R.R. Generalized orthopair fuzzy sets. IEEE Trans. Fuzzy Syst. 2016, 25, 1222-1230. [CrossRef]

45. Xiao, F.; Ding, W. Divergence measure of Pythagorean fuzzy sets and its application in medical diagnosis. Appl. Soft Comput. 2019, 79, 254-267. [CrossRef]

46. Begum, S.S.; Srinivasan, R. Some properties on intuitionistic fuzzy sets of third type. Ann. Fuzzy Math. Inform. 2015, 10, 799-804.

47. Son, L.H.; Cuong, B.C.; Lanzi, P.L.; Thong, N.T. A novel intuitionistic fuzzy clustering method for geo-demographic analysis. Expert Syst. Appl. 2012, 39, 9848-9859. [CrossRef]

48. Yang, Y.; Chiclana, F. Intuitionistic fuzzy sets: Spherical representation and distances. Int. J. Intell. Syst. 2009, 24, 399-420. [CrossRef]

49. Maji, P.K.; Biswas, R.; Roy, A.R. Intuitionistic fuzzy soft sets. J. Fuzzy Math. 2001, 9, 677-692. [CrossRef]

50. Cornelis, C.; De Cock, M.; Kerre, E.E. Intuitionistic fuzzy rough sets: At the crossroads of imperfect knowledge. Expert Syst. 2003, 20, 260-270. [CrossRef]

51. Zhu, B.; Xu, Z.; Xia, M. Dual hesitant fuzzy sets. J. Appl. Math. 2012, 2012, 879629. [CrossRef]

52. Alkouri, A.; Salleh, A. Complex intuitionistic fuzzy sets. In AIP Conference Proceedings; Ari-Wahjoedi, B., Razali, R., Narahari, M., Eds.; AIP: Melville, NY, USA, 2012; Volume 1482, pp. 464-470. [CrossRef]

53. Radojević, D. Logical aggregation based on interpolative Boolean algebra. Mathw. Soft Comput. 2008, 15, $125-141$.

54. Milošević, P.; Poledica, A.; Rakićević, A.; Dobrić, V.; Petrović, B.; Radojević, D. IBA-based framework for modeling similarity. Int. J. Comput. Intell. Syst. 2018, 11, 206-218. [CrossRef]

55. Gottwald, S. Many-valued logics. In Philosophy of Logic; North-Holland: Amsterdam, The Netherlands, 2007; pp. 675-722. [CrossRef]

56. Hájek, P.; Godo, L.; Esteva, F. A complete many-valued logic with product-conjunction. Arch. Math. Logic 1996, 35, 191-208. [CrossRef]

57. Brown, M. Boolean Reasoning: The Logic of Boolean Equations, 2nd ed.; Dover Publications: Mineola, NY, USA, 2003.

58. Milošević, P. IFS-IBA Approach: Interpolative Boolean Algebra in Intuitionistic Fuzzy Set Theory. Ph.D. Thesis, University of Belgrade, Belgrade, Serbian, 2017.

59. Nison, S. Japanese Candlestick Charting Techniques: A Contemporary Guide to the Ancient Investment Techniques of the Far East, 2nd ed.; Prentice Hall: Upper Saddle River, NJ, USA, 2001.

60. Nešić, I.; Milošević, P.; Rakićević, A.; Petrović, B.; Radojević, D. Modeling candlestick patterns with interpolative Boolean algebra for investment decision making. In Soft Computing Applications, Proceedings of 5th International Workshop Soft Computing Applications (SOFA), Szeged, Hungary, 22-24 August 2012; Balas, V.E., Fodor, J., Várkonyi-Kóczy, A.R., Dombi, J., Jain, L.C., Eds.; Springer: Berlin/Heidelberg, Germany, 2013; pp. 105-115. [CrossRef]

61. Marszałek, A.; Burczyński, T. Modeling and forecasting financial time series with ordered fuzzy candlesticks. Inform. Sci. 2014, 273, 144-155. [CrossRef]

62. Naranjo, R.; Arroyo, J.; Santos, M. Fuzzy modeling of stock trading with fuzzy candlesticks. Expert Syst. Appl. 2018, 93, 15-27. [CrossRef] 